\section{Cardiovascular fibrosis, hydrocephalus, ophthalmoplegia, and visceral involvement in an American child with Gaucher disease}

EDITOR-We have recently performed the molecular characterisation of the glucocerebrosidase alleles of a child with an unusual presentation of type 3 Gaucher disease. This patient was among the first described cases of Gaucher disease with cardiac involvement, oculomotor abnormalities, and hydrocephalus, ${ }^{1}$ features that were subsequently described in patients from Japan, ${ }^{23}$ Israel, ${ }^{4}$ Spain, ${ }^{56}$ Turkey, ${ }^{78}$ Canada, $^{9}$ and the USA ${ }^{10}{ }^{11}$ (table 1). Interestingly, many of these patients were reported to be homozygous for the mutation $\mathrm{D} 409 \mathrm{H}$ in the human glucocerebrosidase gene. Similar to the latest case with genotype $\mathrm{D} 409 \mathrm{H} / \mathrm{D} 409 \mathrm{H}$ described in this journal by Chabás et $a l,{ }^{5}$ our patient had early and aggressive visceral disease. However, unlike many of the other cases reported, pathological evaluation showed cardiovascular fibrosis, rather than calcification, and Gaucher cells were found scattered within the areas of fibrosis.

This child was diagnosed at 6 months of age with splenomegaly and abnormal saccadic eye movements were observed at 1 year of age. At 6 years of age, he was noted to have delayed growth (height and weight less than the $1 \mathrm{st}$ centile), scattered ecchymoses, a thoracolumbar gibbus deformity, and a grade 3/6 systolic ejection murmur. His liver extended $4 \mathrm{~cm}$ below the right costal margin, and his spleen was palpable at the left pelvic brim and extended across the midline. A neuro-ophthalmological evaluation disclosed an ophthalmoplegia with absent saccadic movements. His gait was broad based, and the muscle tone was decreased in his lower extremities, with symmetrically hyperactive patellar and ankle reflexes. Laboratory evaluation was significant for a haemoglobin level of $10.1 \mathrm{~g} / \mathrm{dl}$ and a platelet count of $105000 / \mathrm{mm}^{3}$. Long bone surveys, chest $x$ rays, an EKG, and an echocardiogram were reported as normal. An EEG was suggestive of mild bilateral cerebral dysfunction, and a cranial CT scan showed a compensated, communicating hydrocephalus. At $6 \frac{1}{2} 2$ years of age, the child developed acute pulmonary oedema. He experienced a respiratory arrest and subsequently a terminal cardiac arrest. ${ }^{1}$

Necropsy showed unexpected cardiac ventricular hypertrophy, intimal fibrosis of the coronary arteries and aorta, and a grey fibrotic thickening of the intimal surface at the root of the aorta. ${ }^{1}$ Gaucher cells were scattered along the intima and media of the aorta in the area of fibrosis. ${ }^{1}$

Mutation analysis, performed by sequencing of DNA, showed that the patient was heterozygous for mutation D409H and a recombinant allele encompassing mutations L444P, A456P, and V460V (RecNciI). Southern blot analysis using the restriction enzymes $S s p \mathrm{I}$ and $S s t \mathrm{II}$ confirmed that this was not a fusion allele. Because both mutant alleles contain sequence changes found in the pseudogene, an NciI restriction digest of a PCR amplified fragment containing exons 8-11 was performed to determine which allele contained the L444P mutation. Analysis of both alleles after $\mathrm{NciI}$ digestion, electrophoretic separation on an agarose gel, excision, and gel purification and sequencing confirmed that the $\mathrm{D} 409 \mathrm{H}$ mutation was not present on the RecNciI allele. The RecNciI allele is most sequencing likely a null allele, since in the

Table 1 Patients with type 3 Gaucher disease and cardiovascular abnormalities

\begin{tabular}{|c|c|c|c|c|c|c|c|c|c|c|}
\hline \multirow[b]{2}{*}{ Report } & \multirow{2}{*}{$\begin{array}{l}\text { Country } \\
\text { of origin }\end{array}$} & \multirow{2}{*}{$\begin{array}{l}\text { No of } \\
\text { cases }\end{array}$} & \multirow[b]{2}{*}{ Age } & \multirow[b]{2}{*}{ Consanguinity } & \multirow[b]{2}{*}{ Genotype } & \multicolumn{5}{|l|}{ Symptoms } \\
\hline & & & & & & Visceral & Neurological & Cardiac & Eye & Other \\
\hline Chabas et al $\overline{5}$ & Spain & 3 sibs & $\begin{array}{l}10 \\
16 \\
17\end{array}$ & No & $\begin{array}{l}\mathrm{D} 409 \mathrm{H} / \\
\mathrm{D} 409 \mathrm{H}\end{array}$ & $\begin{array}{l}\text { Mild } \\
\text { Moderate }\end{array}$ & $\begin{array}{l}\text { Abnormal saccades, } \\
\text { one had tonic/clonic } \\
\text { seizures }\end{array}$ & $\begin{array}{l}\text { Calcifications of aortic } \\
\text { and mitral valves and } \\
\text { ascending aorta }\end{array}$ & $\begin{array}{l}\text { Corneal } \\
\text { opacities }\end{array}$ & NA \\
\hline $\begin{array}{l}\text { Abrahamov et } \\
a l^{4}\end{array}$ & Israel & $\begin{array}{l}12 \text { pts in } \\
3 \\
\text { families }\end{array}$ & $2-20$ & Yes & $\begin{array}{l}\text { D409H/ } \\
\text { D409H }\end{array}$ & Mild & $\begin{array}{l}\text { Absent saccades, } \\
\text { mild dilatation of } \\
\text { lateral ventricles }\end{array}$ & Calcification of valves & $\begin{array}{l}\text { Corneal } \\
\text { opacities }\end{array}$ & NA \\
\hline Sharratt $e t a l^{9}$ & Canada & 1 & 12 & Yes & NA & Moderate & NA & $\begin{array}{l}\text { Calcifications of } \\
\text { mitral and aortic } \\
\text { valves and left atrium }\end{array}$ & NA & NA \\
\hline Saraclar $e t a l^{8}$ & Turkey & 2 sibs & $\begin{array}{l}15 \\
9\end{array}$ & Yes & NA & Mild & NA & $\begin{array}{l}\text { Mitral and aortic } \\
\text { valve involvement }\end{array}$ & NA & NA \\
\hline Uyama et $a l^{23}$ & Japan & 3 sibs & $\begin{array}{l}42 \\
35 \\
27\end{array}$ & No & $\begin{array}{l}\mathrm{D} 409 \mathrm{H} / \\
\mathrm{D} 409 \mathrm{H}\end{array}$ & Mild & $\begin{array}{l}\text { Abnormal saccadic } \\
\text { eye movements, } \\
\text { leptomeningeal } \\
\text { fibrosis }\end{array}$ & $\begin{array}{l}\text { Mitral and aortic } \\
\text { valve calcification, } \\
\text { intimal fibrosis of } \\
\text { ascending aorta }\end{array}$ & $\begin{array}{l}\text { Corneal } \\
\text { opacities }\end{array}$ & $\begin{array}{l}\text { Azoospermia, } \\
\text { progressive hearing } \\
\text { loss, deformed toes, } \\
\text { hyperextensible skin, } \\
\text { hernias }\end{array}$ \\
\hline Erduran et al & Turkey & 1 & 12 & Yes & NA & Mild & $\begin{array}{l}\text { Communicating } \\
\text { hydrocephalus, } \\
\text { choreoathetoid } \\
\text { movements of head }\end{array}$ & $\begin{array}{l}\text { Left ventricular } \\
\text { hypertrophy }\end{array}$ & $\begin{array}{l}\text { Corneal } \\
\text { opacities }\end{array}$ & $\begin{array}{l}\text { Clinodactyly, } \\
\text { sensorineural hearing } \\
\text { loss }\end{array}$ \\
\hline Beutler et $a l^{10}$ & $\begin{array}{l}\text { Britain/ } \\
\text { Germany }\end{array}$ & 1 & 18 & Yes & $\begin{array}{l}\mathrm{D} 409 \mathrm{H} / \\
\mathrm{D} 409 \mathrm{H}\end{array}$ & Moderate & $\begin{array}{l}\text { Hydrocephalus, } \\
\text { oculomotor apraxia }\end{array}$ & "Cardiac disease" & NA & NA \\
\hline $\begin{array}{l}\text { This report } \\
\text { and Wilson } \\
\text { et } a l^{1}\end{array}$ & USA & 1 & 6 & No & $\begin{array}{l}\mathrm{D} 409 \mathrm{H} / \\
\text { recombinant } \\
\text { allele }\end{array}$ & Severe & $\begin{array}{l}\text { Absent saccades, } \\
\text { mild communicating } \\
\text { hydrocephalus }\end{array}$ & $\begin{array}{l}\text { Ventricular } \\
\text { hypertrophy, aortic } \\
\text { and coronary artery } \\
\text { intimal fibrosis }\end{array}$ & NA & NA \\
\hline Casta $e t a l^{11}$ & USA & 1 & 15 & NA & NA & Mild & NA & $\begin{array}{l}\text { Calcification of } \\
\text { ascending aorta, aortic } \\
\text { and mitral valves }\end{array}$ & $\begin{array}{l}\text { Corneal } \\
\text { deposits }\end{array}$ & NA \\
\hline
\end{tabular}


homozygous state it has only been observed in cases of Gaucher disease presenting as hydrops fetalis. ${ }^{12}$

Thus, our molecular findings in this young child with Gaucher disease provide strong support that the unusual manifestations of aortic and coronary artery involvement, hydrocephalus, and absent saccadic movements are associated with the $\mathrm{D} 409 \mathrm{H}$ mutant allele, as observed in other cases. $^{2-5} 10$ However, since severely affected patients with Gaucher disease do not generally present with the cardiovascular complications and hydrocephalus described here, it is unlikely that these unusual findings are solely the result of glucocerebrosidase deficiency. A more extensive characterisation of genes surrounding glucocerebrosidase, ${ }^{13}$ as well as other genetic and environmental factors, may help to explain the aetiology of this phenotype.

DEBORAH L STONE NAHID TAYEBI

CHANELLE COBLE

EDWARD I GINNS

Clinical Neuroscience Branch, National Institute of Mental Health, National Institutes of Health, Bldg 49, Room B1EE16, 49 Convent Drive, MSC4405, Bethesda, MD 20892-4405, USA

Correspondence to: Dr Sidransky, sidranse@irp.nimh.nih.gov

1 Wilson ER, Barton NW, Barranger JA. Vascular involvement in type 3 neuronopathic Gaucher's disease. Arch Pathol Lab Med 1985;109:82-4.

2 Uyama E, Uchino M, Ida H, Eto Y, Owada M. D409H/D409H genotype in Uyama E, Uchino M, Ida H, Eto Y, Owada M. D
Gaucher-like disease. $\mathcal{F}$ Med Genet 1997;34:175.
3 Uyama E, Takahashi K, Owada M, Okamura R, Naito M, Tsuji S, Kawasaki $\mathrm{S}$, Araki S. Hydrocephalus, corneal opacities, deafness, valvular heart disease, deformed toes and leptomeningeal fibrous thickening in adult siblings: a new syndrome associated with glucocerebrosidase deficiency and a mosaic population of storage cells. Acta Neurol Scand 1992;86:407-20.

4 Abrahamov A, Elstein D, Gross-Tsur V, Farber B, Glaser Y, Hadas-Halpern I, Ronen S, Tafakjdi M, Horowitz M, Zimran A. Gaucher's disease variant characterised by progressive calcification of heart valves and unique genotype. Lancet 1995;346:1000-3.

5 Chabás A, Gort L, Montfort M, Castelló F, Dominguez MC, Grinberg D, Vilageliu L. Recurrence of the D409H mutation in Spanish Gaucher disease patients: description of a new homozygous patient and haplotype analysis. F Med Genet 1998;35:775-7.

6 Chabás A, Cormand B, Grinbert D, Burguera JM, Balcells S, Merino JL, Mate I, Sobrino JA, Gonzalez-Duarte R, Vilageliu L. Unusual expression of Gaucher's disease: cardiovascular calcifications in three sibs homozygous for the $\mathrm{D} 409 \mathrm{H}$ mutation. F Med Genet 1995;32:740-2

7 Erduran E, Mocan H, Gedik Y, Kamaci R, Ökten A, Değer O. Hydrocephalus, corneal opacities, deafness, left ventricle hypertrophy, clinodactyly in lus, corneal opacities, deafness, left ventricle hypertrophy, clinodactyly in deficiency. Genet Couns 1995;6:211-15.

8 Saraçlar M. Atalay S, Koçak N, Özkutlu S. Gaucher's disease with mitral and aortic involvement: echocardiographic findings. Pediatr Cardiol 1991;13:56-8.

9 Sharratt GP, Price D, Curtis JA, Cornel G. Gaucher's disease with mitral valve calcification. Pediatr Cardiol 1992;13:127-8

10 Beutler E, Kattamis C, Sipe J, Lipson M. 342 mutation in Gaucher's disease. Lancet 1995;346:1637.

11 Casta A, Hayden K, Wolf WJ. Calcification of the ascending aorta and aortic and mitral valves in Gaucher's disease. Am F Cardiol 1984;54:1390-1.

12 Tayebi N, Reissner KJ, Lau EK, Stubblefield BK, Klineburgess AC, Martin $\mathrm{BM}$, Sidransky E. Genotypic heterogeneity and phenotypic variation among patients with type 2 Gaucher's disease. Pediatr Res 1998;43:571-8.

13 Sidransky E, Ginns EI. Gaucher disease plus. F Med Genet 1997;34:876-9.

14 Winfield SL, Tayebi N, Martin BM, Ginns EI, Sidransky E. The identification of three additional genes contiguous to the glucocerebrosidase locus on chromosome Pq21. Implications for Gaucher disease. Genome Res 1997;7:1020-6. 